\title{
ARE WE READY FOR A CHRONIC CRISIS? REFLECTIONS ON THE EXPERIENCE OF TEACHING DURING CONFINEMENT
}

\author{
I. Tort-Ausina1 ${ }^{1}$, A. Vidaurre ${ }^{1}$, J. Riera1, M.A Gámiz-González², J.M. Meseguer- \\ Dueñas $^{1}$, J.A. Gómez-Tejedor ${ }^{1}$, S. Quiles-Casado1, Maria-Antonia Serrano1, \\ J. Molina-Mateo ${ }^{1}$ \\ ${ }^{1}$ Universitat Politècnica de València (SPAIN) \\ ${ }^{2}$ Universidad Internacional de Valencia (SPAIN)
}

\begin{abstract}
The lockdown period that started on March 2020 due to the COVID-19 pandemic was a big challenge for students and teachers. Technology made the continuation of the teaching easier, and it also allowed teachers to keep the contact both with colleagues and with students. New teaching and evaluation methodologies and new coordination and cooperation systems had to be quickly implemented. Our previous experience in flipped teaching, active methodologies and validation of online exams, helped us to tackle the transition from face-to-face to fully online teaching. The experience was satisfactory for the theory classes, but some problems in the laboratory classes have emerged.
\end{abstract}

After that, right now, some extra work is needed to overcome these difficulties considering that maybe the situation would become chronic. Once that we got over the shock of the first moment, the question is whether the resilience mechanisms will remain, and if the hybrid teaching (face-to-face \& online) can replace effectively face-to-face teaching for a long time. In this work we present the students' opinion on this question. The analysis has been performed at the School of Design Engineering of the Universitat Politècnica de València, in different courses of physics of several engineering degrees (all of them, first academic year courses).

One example of good practice during confinement was the weekly coordination meetings between teachers of the same course and between teachers of physics courses in different degrees. Another one was the online exams, taking advantage of the automatic correction tests, and including images of the documents written by the students for later review.

The academic results were comparable to those of a normal course and student and teachers' surveys were conducted to know their opinion. The good performance during this new situation was due to the collaboration, patience, understanding and trust of the students and teachers that was created in those moments of crisis.

Students' opinion on the online teaching during their lockdown was positive. This work shows this students' opinion on the methodology used during the lockdown, and the process from previous flipped teaching methodologies experiences to a completely online teaching. At the same time, the students' opinion on the laboratory practices was also analysed.

Finally, this work also shows the reflection of the involved teachers on the additional workload and on the capacity for cooperation and communication between them, during the lockdown period and the subsequent year.

After gathering the opinion of teachers and students, we can conclude that the situation of the pandemic has been addressed with reasonable success. Performance of students is not affected, the theoretical classes have been developed normally, while the practical classes seem to need some changes to be adapted to the new situation as far as possible. Given that this situation persists, it is useful to carry out this type of study to be able to adapt teaching to future circumstances.

Keywords: confinement, face to face teaching, online teaching, students' opinion.

\section{INTRODUCTION}

On $11^{\text {th }}$ March 2020, the World Health Organization (WHO) declared the COVID-19 (coronavirus disease 2019) caused by the 2019 novel coronavirus (2019-nCoV) a pandemic. This has brought radical changes in all aspects of our lives. The most significant was the social distancing and restrictive movement that markedly affected the traditional educational practices. 
The flipped teaching (FT) is a widely implemented methodology that has demonstrated to be very effective in high education, mainly in STEAM subjects [1]. It combines a number of student-centred activities including, among others, peer assisted learning, cooperative learning, problem-based learning and gamification [2]. Students have access to learning resources like articles, pre-recorded videos, and YouTube links before the class. The online classroom time is then used to deepen understanding through discussion with faculty and peers. This is a very effective way of encouraging skills such as problem-solving, critical thinking and self-directed learning. Some authors have shown that adding educational gaming elements improves the classroom dynamics, increase students' participation and interest in online preparation before class [3], [4].

Although many papers show that the academic results improve when Flip Teaching (FT) is applied [5], [6], some authors have indicated that, in the case of first-year students, a transition between the dominant transmission model of teaching is needed to engage first year student in FT [7]. When students have been asked, some of them say that they prefer the combination of flip teaching with a traditional methodology [8], [9].

The term virtual flipped classroom was introduced by Ismail and Abdulla in 2018 as a combination of Flipped Classroom and Virtual Classroom. They applied the virtual to a group of students to some selective topics of a computer programming course. They found a significant difference in learning achievement and motivation after applying the virtual flipped classroom.

The implementation of the FT in the classroom has always been accompanied by different teaching tools that facilitate the work of students out of class to prepare the class seasons (videos, documents, self-evaluation exams, tasks, and communication tools. All these strategies and tools have been based on the technologies of the information and communication (ICT). In this work, the material was made available to students through the Universitat Politècnica de València's online teaching platform, PoliformaT ("PoliformaT," 2003), adapted from Sakai ("Sakai Learning Management System," 2018). These tools were used for the realization of both collective and individualized tutoring, examination review, cooperative work and oral presentations.

The lockdown forced to move to online teaching. Unified communication and collaboration platforms like Microsoft Teams, Google Classroom, Zoom, were quickly introduced and became common for communication between teachers and students. These tools were used for both, collective and individualized communication, as well as for examination review, cooperative work and oral presentations.

In this work, we present the effect of the lockdown on the academic results and students' perception. The analysis has been performed at the School of Design Engineering of the Universitat Politècnica de València, to students of the Degree on Electronic Engineering and Industrial Automation (DEEIA) and of the Degree on Aerospace Engineering (DAE).

\section{METHODOLOGY}

This work was carried out for two different groups enrolled in physics courses (physics and electromagnetism) of two degrees, DAE and DEEIA, respectively. Physics of DEA, with 114 students in two groups, is annual whereas electromagnetism of DEEIA, with 152 students in three groups, is a second semester course. Prior to the lockdown, the first half of the course of DAE had been face-toface classes and the second part was online. In the case of DEEIA, less than one-third of the course was face-to-face and the rest of the course was online.

Both courses applied the FT methodology. The students had different materials to prepare the class sessions: videos, documents, and online exams. The students worked in teams preparing the lab reports, solving problems or evaluating the problems solved by another team. During the class sessions, apart from some explanations of the most difficult concepts, some sessions were dedicated to solving problems in groups, and, in another session, students had to present the solved problems. Continuous assessment with different tasks and exams was programmed along the course. Some gamification activities, as Kahoot were also used [10].

Once the lockdown was declared, the class sessions were online (by Microsoft Teams) and synchronous. All the sessions were recorded and all the materials were available for students. The programmed tasks and exams were kept with the same calendar, but the oral presentation and the exams were online. The online exams consisted of numerical problems that students had to solve (students had different problems and/or different data). The students had to write the numerical answers 
that were automatically corrected by the exams tool and they also had to upload a file with the scanned images of their resolution. Then, if the student asked, the procedure, and not only the numerical response could be considered. Regarding the lab sessions, the data collected by students of the previous year were given to the students. They had to perform the corresponding graphs and calculations to get the asked results.

The communication with the teacher was made by different online ways: email, chat or videoconference. Before the lockdown most of the students used to communicate using the same tools, except the videoconference. Students had to share documents and maintain online meetings to complete the tasks as a team.

A post-course survey was delivered to measure students' perception. 120 students (61 from DAE and 59 from DEEIA) completed the survey that consisted of 5 -point Likert-type questions and 5 open questions. Students rated statements about the course where 1 is 'Strongly disagree' and 5 is 'Strongly agree' and also 1 is 'very bad' and 5 is 'excellent'. They were also asked about the number of hours per week they dedicated to the course. The questions were related with the course (7 questions) and also with the locked down situation (4 questions).

\section{RESULTS}

\subsection{Survey on the students' opinion about the teaching experience during the lock down}

The starting point of our reflections on the recent experience on online teaching, caused by the lock down because of the COVID-19, was that our previous experience both in active methodologies and in FT was going to be an important advantage to afford the situation that had arisen.

In the one hand, the results of the opinion survey show that the students have valuated positively the adaptation to the online teaching. When the students of the Degrees on Aerospace Engineering (DAE) and of the Degree on Electronic Engineering and Industrial Automation (DEEIA) where asked about the teaching experience during confinement, most of them ( $82 \%$ and $78 \%$ respectively) say that despite the limitations of the situation we were going through, the course had managed to function properly (Figure 1).

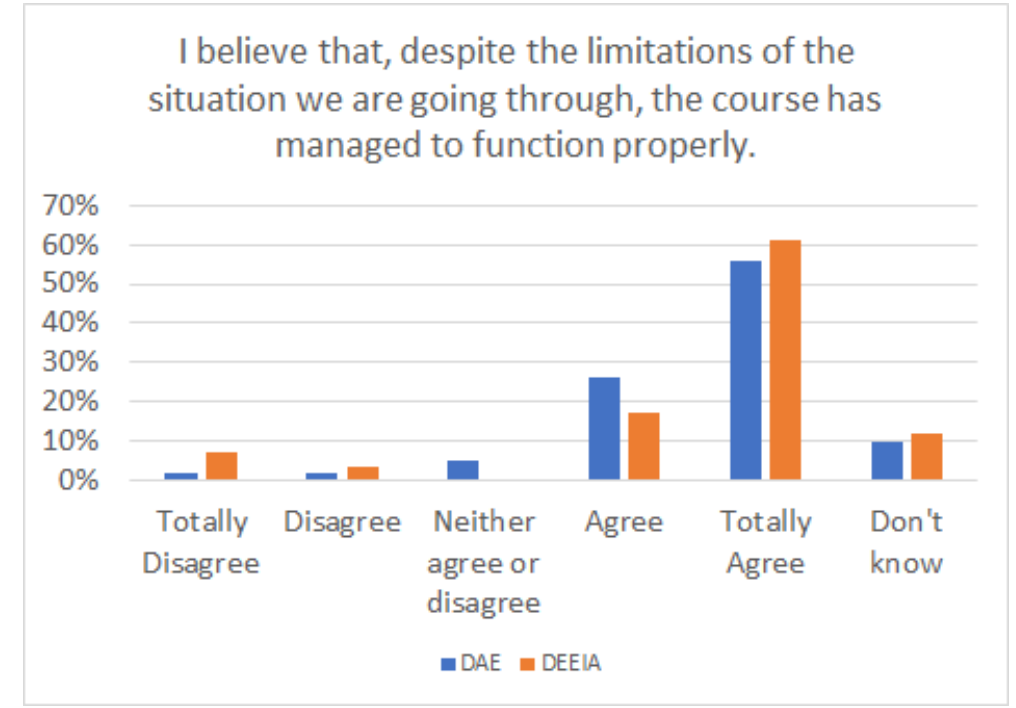

Figure 1: Results of the general question about the functioning of the course.

On the other hand, when students were asked about their work, $64 \%$ and $73 \%$ of them, respectively, declare that they agree or fully agree on the fact that they have been able to follow the course reasonably well (Figure 2).

This success of our work developed in the course has been a consequence of the fast adaptation to the new and suddenly happened situation, in an extremely short period of time, with right and wrong aspects, but above everything of the previous work made in the frame of the active methodologies and FT. 


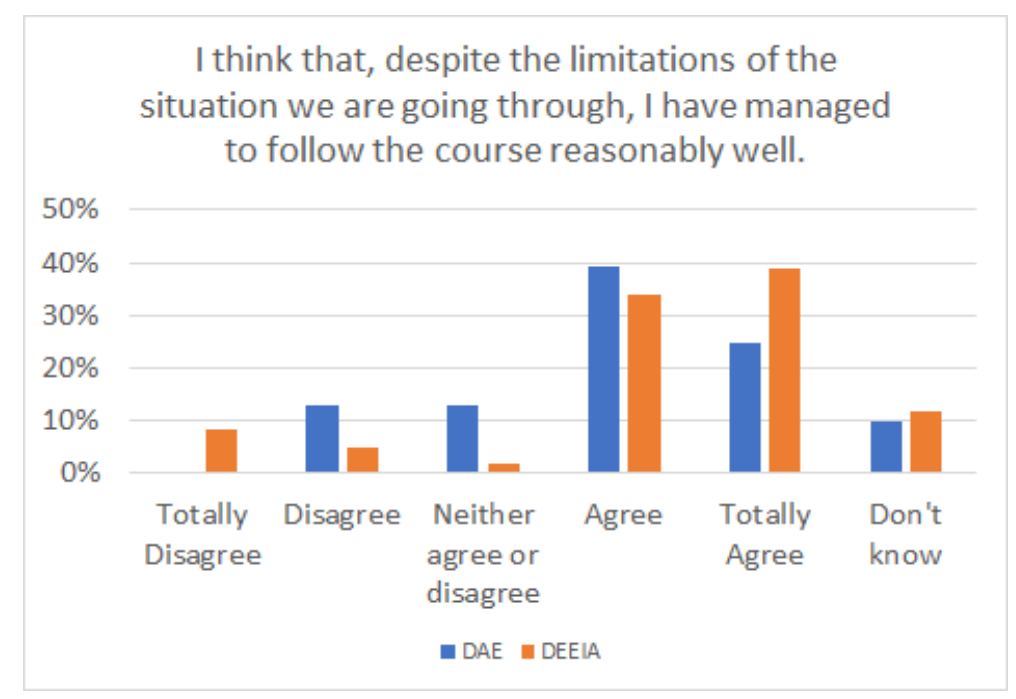

Figure 2: Results of the question about the work made by the students.

Keeping the uncertainty of the near future in mind, as clearly none of us knows how the situation will exactly be for the next academic year, the crisis we are being through is showing as a likely scenario where face to face and online teaching and synchronous and asynchronous training will coexist, as the main features of the university education of the future. Despite how forced and unexpected the change in our teaching methodologies has been, we think that this experience should became an opportunity to take benefit from the actual situation to act in this sense for the future.

Some teaching tools, such as videoconference, cooperative working or gamification have been proved to be solid and useful ones, even when we were not familiar with them and we had to learn how to use them and how to implement them in our courses suddenly, quickly, and with no other option. Our general impression now is that most of these tools have come to stay. The students' opinion that is shown in this communication, gathered after a term of teaching using all these tools (Figure 1) shows that it has been a successful experience for them.

Furthermore, as the FT has taken so much prominence, the opinion of the students on it was also very important for us. To start, our experience supports the previous results obtained in literature showing that flipped teaching improves significantly, but not radically, the academic performance of our students [11], [12]. However, FT is still having a negative image according to the first year students [7], [13] and this is also supported by our results. Even within the context of the lock down, in which, as already mentioned, their opinion on the management and development of the course was positive, when they were asked about the interest of the course itself and about the FT their opinion shows a very high interest on the course, and many objections to the methodology (Figures 3 and 4).

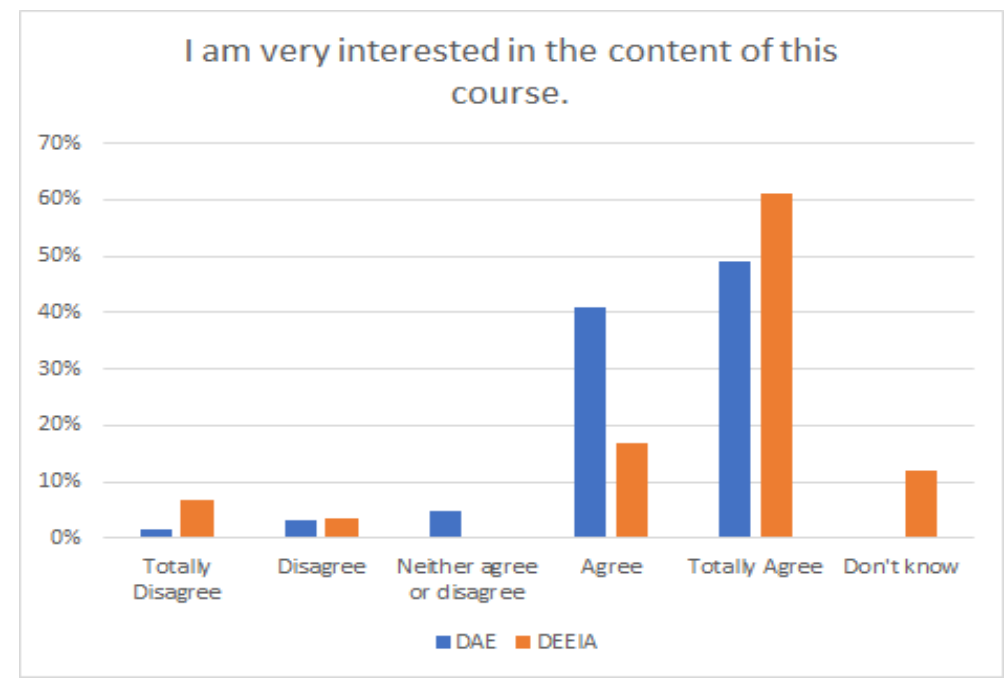

Figure 3: Results of the question about their interest on the course. 


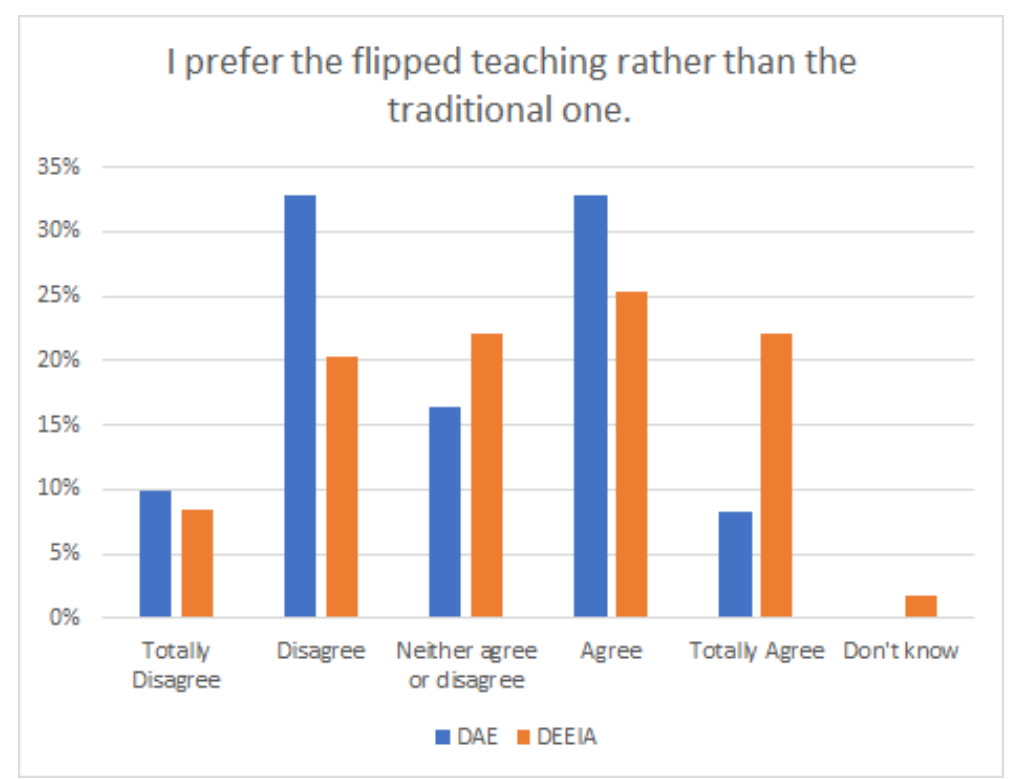

Figure 4: Results of the question about their preference between flipped teaching or traditional teaching

This objection to the FT is especially evident when we ask them if they would extend the methodology model to other subjects (Figure 5)

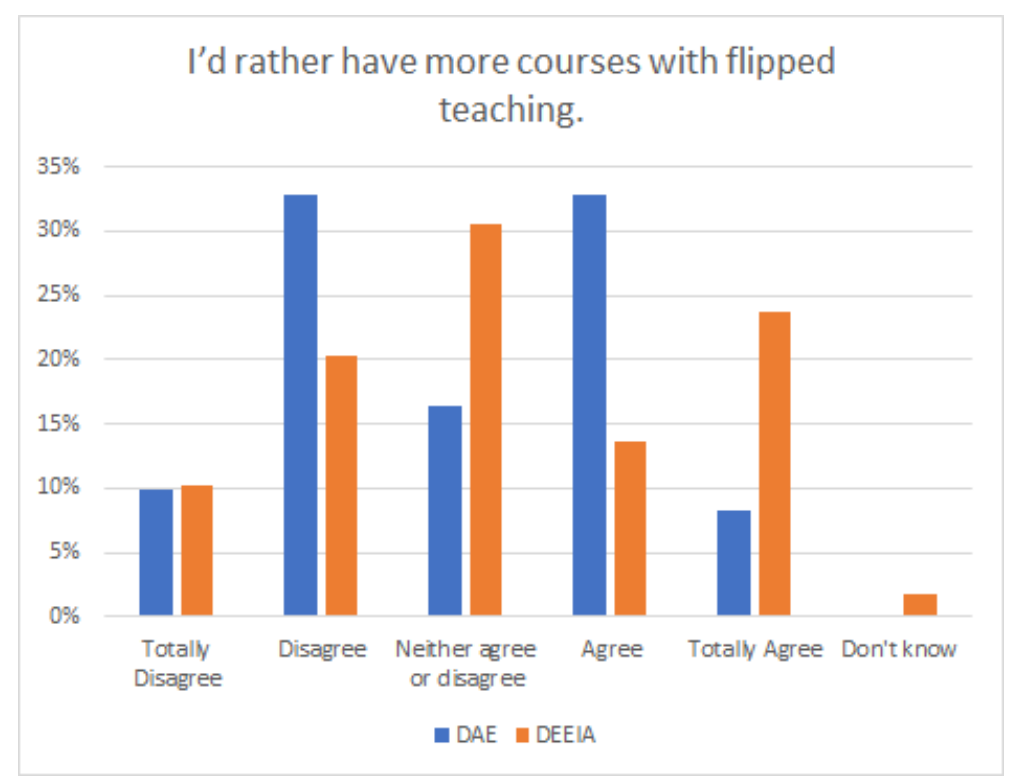

Figure 5: Results of the question about the possibility of having more courses with flipped teaching.

These last answers contrast with their opinion when asked separately about each one of the components used in FT. As an example, Figure 6 shows the results on the assessment of some of the activities carried out and materials used by students. 


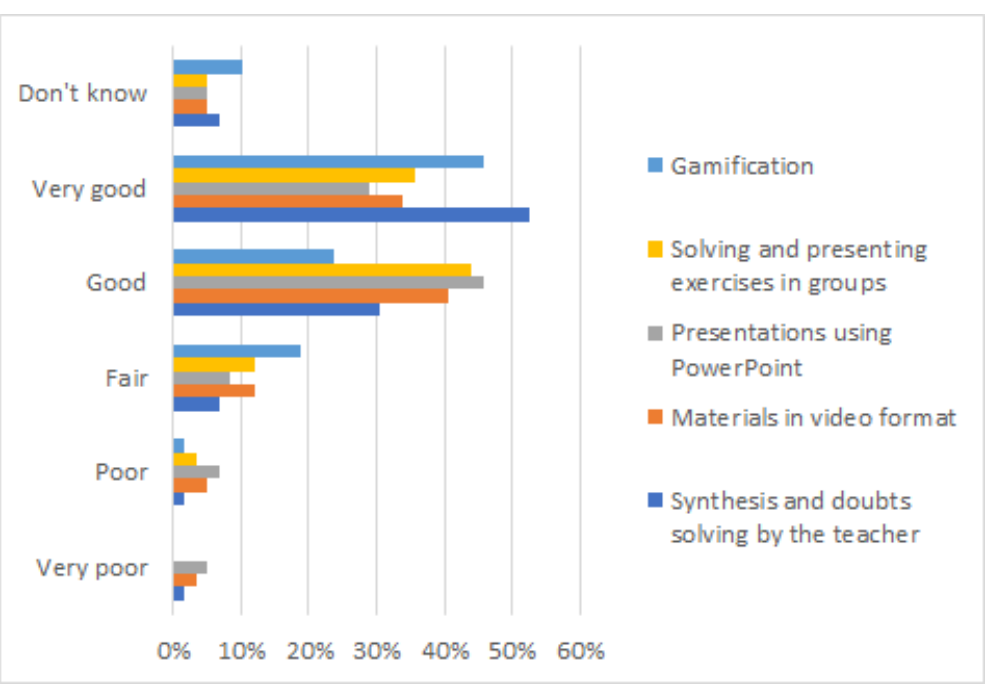

Figure 6: Results of the question about the different tools and materials used.

One of the teaching activities where a deep reflection is needed to find good solutions in a context or emergency (or even in a possible hybrid future teaching model) are the laboratory practices. In the previous years, we had been using flipped teaching at the lab, where all the introduction was explained through videos that the student should see before arriving to the laboratory session, and the time of the session was dedicated to the experimental data collection and analysis. However, this previous experience was not transferable to the lock down, because the instrumental and experimental part was of utmost importance in our lab practices (in subjects such as Mechanics or Electricity).

The students' opinion confirms this last idea: the laboratory practices are the lowest rated activity by the students (Figure 7). Then, new models have to be explored to make the activity at the lab compatible with the new situation in a hybrid teaching, in which collaborative and synchronous work of students could be compatible with the security conditions at the labs. Maybe simulations could be introduced in the future, when possible, to perform online lab practices.

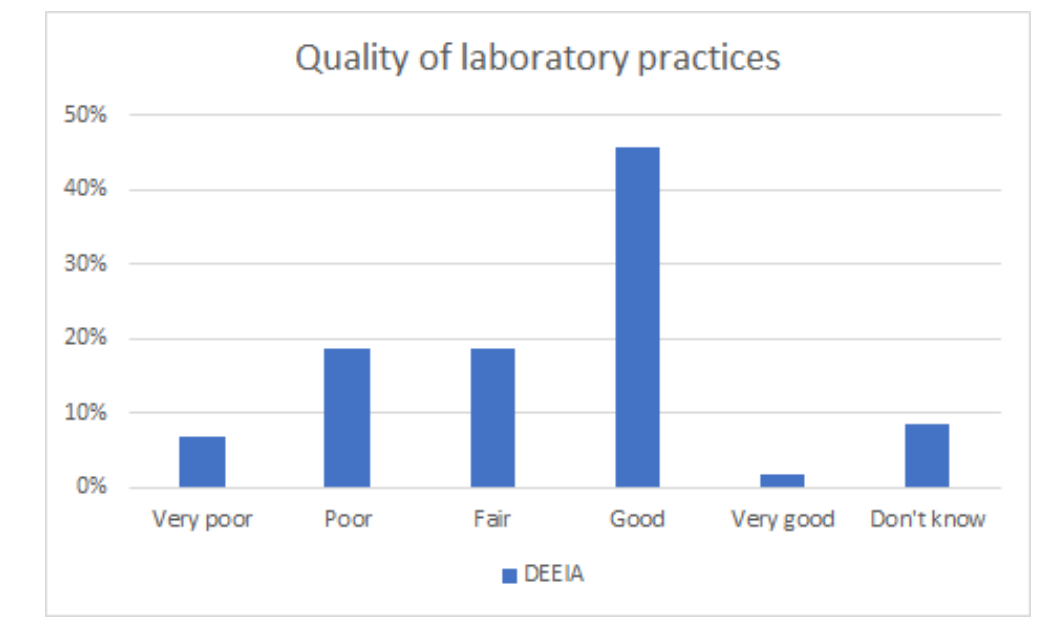

Figure 7: Results of the question about the quality of the laboratory practices

\subsubsection{Students' opinions: results of open-ended questions.}

There is a high number of responses, since in both degrees around 45 students have responded out of the total that completed the survey.

Both DAE and DEEIA students positively valued the interest of the teachers, as well as the activities proposed (inside and outside the classroom) that helped them to follow the class online, encouraged their participation and aroused their interest, overcoming the problem of online teaching.

The students quite unanimously valued positively the content of the programme, the structuring of contents in the Poliformat platform, the exercises solved in class, the team problem solving, and the 
active participation with the different proposed activities (tasks, dialogues, monologues, Kahoots). They also valued the material hosted on the web (videos, presentations, problems).

The main complaints were focused on the laboratory work (they did not find sense to work with data that they had not measured). In addition, students clearly indicated that they were not in favour of flipped teaching for this subject. They think that classes should be taught with more detailed theoretical explanations and with a greater number of exercises solved by the teacher. They also thought that the required number of tasks was excessive, especially during confinement and suggested spending less time on complementary activities to devote to reviewing the theory. However, several students indicated that during online teaching the explanations had been more detailed and the resolution of exercises clearer, compared to pre-confinement teaching.

Regarding the assessment that students made of their work, in general they thought they have done an effort and a good job in the course, adapting well to online teaching. Several students commented they had connection problems, that they had to share the computer, or that the study space was not adequate. These problems caused them to work less than usual. Some indicated that they had to work harder since it was difficult for them to concentrate, while others had not noticed much change or adapted well. Several students commented they had personal or even mental health problems caused by the emotional situation due to confinement that made work difficult. In general, they indicated having missed the study environment of the class.

\subsection{Reflections on our work: Two good practices with a positive result}

Good practices in the field of education are identified as experiences that have been implemented with positive results, being effective in a specific context, contributing to the solution of specific problems, and generally achieving an educational improvement. In this section, we describe two examples of actions that we have carried out, and that are considered to meet the beforementioned characteristics.

\subsubsection{Online exams with images for review}

Before the pandemic, our working group had been using a tool to carry out on-line exams with automatic correction. This Poliformat tool allows teachers to create personalized exams by combining batteries of questions that also provide the possibility of customizing the grade for each exam. The system performs an automatic correction and calculates the student's grade if their answer is within the range of the correct answer. Before the pandemic, this tool was used to take an exam at the end of each lesson in order to be able to carry out a more continuous assessment of the course. Since the automatic correction system does not evaluate the procedures of the students when solving the problems of the exams, for every two automated exams a traditional written exam was carried out in which the procedures were evaluated. In this evaluation system, the traditional written exams had a weight between $50 \%$ and $70 \%$ of the evaluation of the subject while the on-line automated correction exams had a weight between $10 \%$ and $15 \%$ in the final mark.

The confinement prevented the realization of face-to-face exams and all exams became online. In this context, it was decided to evaluate the part of written exams also through the same online exams in such a way that these came to have a weight between $65 \%(50 \%+15 \%)$ and $80 \%(70 \%+10 \%)$ of the total evaluation. Given that online exams only assess the final result without taking into account the procedures, a section was enabled in each exam where the student had to upload a document with the image of the resolution of the problems. If the numerical answer of the automatic correction was correct, the student obtained the maximum mark. If the numerical answer was not correct, the student could ask the teacher to review the procedures in order to assign a proportional grade in a correction similar to that of a traditional written exam. In this way, it was possible to take an online exam whose assessment is more similar to that of a written exam where the resolution procedures are assessed.

In this way, it was possible to combine the advantages of the automated exam, such as the fact that each student had his/her own personalized test, with the advantage of being able to assess the resolution procedures in a more traditional way. The fact that the automated correction already marked the correct answers allowed teachers to focus only on the correction of doubtful cases, what lightened their work.

In summary, the existence of online exams, facilitates the continuous work of students in the courses, by allowing continuous evaluation. However, the need to automate the correction can result in an incomplete assessment, by focusing exclusively on the correct numerical answer. The need to evaluate on-line in times of pandemic has made it necessary to explore other solutions. The use of the image of 
the exam together with its automatic correction, allows us not to depend exclusively on the numerical result, by allowing us to review the methodology of the resolution.

\subsubsection{Regular coordination meetings between teachers}

In the usual operating practice of our working group, the coordination meetings had a frequency of three or four per course, without dates fixed in advance and marked by the preparation of contributions to congress, articles or projects. In each course in which we participated, coordination meetings between the teaching staff were frequent, setting the program, rhythm, evaluation or activities, but without a general operating rule, given the disparity of schedules, and therefore the difficulty in agreeing on dates for the meetings.

After a few initial moments of disorientation and alarm at the beginning of the COVID-19 pandemic, everything has been much easier thanks to the permanent contact between colleagues who share the same course and subject. We had course meetings scheduled weekly at a fixed time from the first moment of confinement, and they were held online through Microsoft Teams, which facilitated agreement on the schedule. Communication also extended outside the meetings, through other online communication channels (WhatsApp, Hangouts, Zoom, Webex, Jitsi ...), which during this period have shown to be extremely useful and essential in daily teaching work. In this way, it was possible to reprogram the contents of the course, the evaluation methodology, create and share new materials, and discover new functionalities of the training platform in a non-face-to-face teaching context. This form of collaborative work allowed to continue with the already made programming of the courses and to keep the synchronous classes as close to the face-to-face ones. The adaptation of the evaluation, also online, anticipated the requirements that the university finally implemented. The academic institution, overwhelmed by the situation, did not collaborate in the entire adaptation process, but did accept all the proposals that were developed as a team. During this period, the difficulties between subjects have become more evident, caused by the lack of communication and transversal coordination between the teaching staff of courses and degrees, related to content, methodology and evaluation.

In the COVID-19 context, the regular meetings had an important emotional component: sharing feelings and sensations has brought the teams of teachers together. Everyone has gone through moments of loneliness and decay, of not understanding the situation or feeling insecure due to the uncertainty of the future. Anyway, everyone also felt satisfied to see that the solutions of some can be shared with others, and that problems are better tackled in a collaborative way. The groups have coalesced.

Based on the result of our experience, it is proposed to extend it to other courses and work groups, with the aim of improving coordination, the exchange of experiences, and the self-evaluation of our work and teaching practice, while, at the same time, increasing the cohesion as a group. This method of fixed periodic meetings (weekly) for the courses, and monthly for the work group, has been permanently implemented in our group.

In summary, we consider that coordination meetings between teachers are an essential tool to improve teaching. Daily tasks and a variety of schedules make continuous planning difficult. The experience acquired during the pandemic with online tools has shown its potential in lightening communication processes and providing them with greater flexibility. This fact indicates the way forward for a normalized post-COVID-19 situation: face-to-face meetings must be combined indistinctly with other on-line ones, thereby allowing a greater frequency of them.

\section{CONCLUSIONS}

In this article, we have analysed and thought about the consequences of the lockdown period due to the COVID-19 pandemic in university education, particularly in the teaching of the physics subject in the first year of the Degrees on Electronic Engineering and Industrial Automation and of the Degree on Aerospace Engineering.

After gathering the students' opinion and teachers' reflections, we can conclude that the situation of the pandemic has been addressed with reasonable success. Our previous experience in flipped teaching, active methodologies, and online exams, helped us to deal with the transition from face-to-face to fully online teaching. In general, the teaching methodology was adapted to an online model with reasonable success, and we were able to apply a methodology like the one before lockdown. Students' opinion about the online teaching during their lockdown was positive, and they have highly valued the adaptation effort made in such a short period of time. Performance of students is not affected, the theoretical 
classes have been developed normally, although some problems in the laboratory classes have yet to be solved.

Given that this situation could persist or could happen again, it is useful to carry out this type of study to be able to adapt our teaching methodologies to future circumstances. We think that many of the teaching tools that we have used in this period, such as web conferencing, cooperative work, or gamification, have proven to be solid and useful, even though we have used them forced by the circumstances and we have learned to use them in a record time. The general impression is that many of these tools are here to stay.

\section{ACKNOWLEDGEMENTS}

Authors would like to thank the Institute of Education Sciences of the Universitat Politècnica de València (Spain) for supporting the Teaching Innovation Group e-MACAFI and for the financial support through PIME Project PIME/2018/B25 and PIME Project PIME/2018/B26.

\section{REFERENCES}

[1] J. O'Flaherty and C. Phillips, "The use of flipped classrooms in higher education: a scoping review. Internet High Educ 25:85-95.," Internet High Educ 2585, vol. 25, pp. 85-95, 2015.

[2] J. Bishop and M. Verleger, "The Flipped Classroom: A Survey of the Research," 120th ASEE Annu. Conf. Expo., pp. 161-178, 2013.

[3] J. Jo, H. Jun, and H. Lim, "A comparative study on gamification of the flipped classroom in engineering education to enhance the effects of learning," Comput. Appl. Eng. Educ., vol. 26, no. 5, pp. 1626-1640, 2018.

[4] A. I. Wang and R. Tahir, "The effect of using Kahoot! for learning - A literature review," Comput. Educ., vol. 149, no. May 2019, p. 103818, 2020.

[5] K. Missildine, R. Fountain, L. Summers, and K. Gosselin, "Flipping the classroom to improve student performance and satisfaction," J. Nurs. Educ., vol. 52, no. 10, pp. 597-599, 2013.

[6] J. A. Gómez-Tejedor et al., "Effectiveness of flip teaching on engineering students' performance in the physics lab," Comput. Educ., vol. 144, p. 103708, Jan. 2020.

[7] L. Tomas, N. Evans, T. Doyle, and K. Skamp, "Are first year students ready for a flipped classroom? A case for a flipped learning continuum," Int. J. Educ. Technol. High. Educ., vol. 16, no. 1, p. 5, Dec. 2019.

[8] J. McCarthy, "Reflections on a flipped classroom in first year higher education," Issues Educ. Res., vol. 26, no. 2, pp. 332-350, 2016.

[9] E. Blair, C. Maharaj, and S. Primus, "Performance and perception in the flipped classroom," Educ. Inf. Technol., vol. 21, no. 6, pp. 1465-1482, 2016.

[10] A. Vidaurre et al., "Students' Opinion About a Teaching Model Based On Team Work, Continuous Formative Evaluation And Flip Teaching Organized Through An E-Learning Platform," EDULEARN2018, pp. 420-428, 2018.

[11] E. Şengel, "To FLIP or not to FLIP: Comparative case study in higher education in Turkey," Comput. Human Behav., vol. 64, pp. 547-555, Nov. 2016.

[12] H.M. Vo, C. Zhu, and N. A. Diep, "The effect of blended learning on student performance at courselevel in higher education: A meta-analysis," Stud. Educ. Eval., vol. 53, pp. 17-28, Jun. 2017.

[13] A.S. Burke and B. Fedorek, "Does 'flipping' promote engagement?: A comparison of a traditional, online, and flipped class," Act. Learn. High. Educ., vol. 18, no. 1, pp. 11-24, 2017. 